\author{
Marquette University \\ e-Publications@Marquette
}

College of Nursing Faculty Research and

Publications

Nursing, College of

7-2021

\title{
Effectiveness of Home Health Care in Reducing Return to Hospital: Evidence from a Multi-hospital Study in the US
}

\author{
Danielle M. Siclovan \\ Froedtert Hospital \\ James Bang \\ St Ambrose University \\ Olga Yakusheva \\ University of Michigan \\ Morris Hamilton \\ Abt Associates \\ Kathleen L. Bobay \\ Loyola University Chicago
}

See next page for additional authors

Follow this and additional works at: https://epublications.marquette.edu/nursing_fac

Part of the Nursing Commons

\section{Recommended Citation}

Siclovan, Danielle M.; Bang, James; Yakusheva, Olga; Hamilton, Morris; Bobay, Kathleen L.; Costa, Linda L.; Hughes, Ronda G.; Miles, Jane M.; Bahr, Sarah J.; and Weiss, Marianne, "Effectiveness of Home Health Care in Reducing Return to Hospital: Evidence from a Multi-hospital Study in the US" (2021). College of Nursing Faculty Research and Publications. 897.

https://epublications.marquette.edu/nursing_fac/897 


\section{Authors}

Danielle M. Siclovan, James Bang, Olga Yakusheva, Morris Hamilton, Kathleen L. Bobay, Linda L. Costa, Ronda G. Hughes, Jane M. Miles, Sarah J. Bahr, and Marianne Weiss 
Marquette University

e-Publications@Marquette

\section{Nursing Faculty Research and Publications/College of Nursing}

This paper is NOT THE PUBLISHED VERSION.

Access the published version via the link in the citation below.

International Journal of Nursing Studies, Vol. 119 (July 2021): 103946. DOI. This article is @ Elsevier and permission has been granted for this version to appear in e-Publications@Marquette. Elsevier does not grant permission for this article to be further copied/distributed or hosted elsewhere without the express permission from Elsevier.

\section{Effectiveness of Home Health Care in Reducing Return to Hospital: Evidence from a Multi-hospital Study in the US}

Danielle M. Siclovan

Froedtert Hospital, 9200 W. Wisconsin Ave., Milwaukee, WI

James T. Bang

Department of Economics, St. Ambrose University, McMullen Hall 124A 518 W. Locust St., Davenport, IA

Olga Yakusheva

University of Michigan School of Nursing, 400 North Ingalls Building, Ann Arbor, MI

Morris Hamilton

Abt Associates, 5001 S Miami Blvd \#210, Durham, NC

Kathleen L. Bobay

Marcella Niehoff School of Nursing, Loyola University Chicago, 2160 S. 1st Ave., Maywood, Illinois

Linda L. Costa

University of Maryland School of Nursing, 655 W. Lombard St., Baltimore, MD

Ronda G. Hughes 
University of South Carolina College of Nursing, 1601 Greene Street, Room 405, Columbia, SC

Jane Miles

Marquette University College of Nursing, PO Box 1881, Milwaukee, WI

Sarah J. Bahr

Marquette University College of Nursing, PO Box 1881, Milwaukee, WI

Marianne E. Weiss

Marquette University College of Nursing, PO Box 1881, Milwaukee, WI

\begin{abstract}
Background

Home health care, a commonly used bridge strategy for transitioning from hospital to home-based care, is expected to contribute to readmission avoidance efforts. However, in studies using disease-specific samples, evidence about the effectiveness of home health care in reducing readmissions is mixed.

\section{Objective}

To examine the effectiveness of home health care in reducing return to hospital across a diverse sample of patients discharged home following acute care hospitalization.

\section{Research design}

Secondary analysis of a multi-site dataset from a study of discharge readiness assessment and post-discharge return to hospital, comparing matched samples of patients referred and not referred for home health care at the time of hospital discharge.
\end{abstract}

\title{
Setting
}

Acute care, Magnet-designated hospitals in the United States

\section{Participants}

The available sample $(n=18,555)$ included hospitalized patients discharged from medical-surgical units who were referred $(n=3,579)$ and not referred $(n=14,976)$ to home health care. The matched sample included 2767 pairs of home health care and non- home health care patients matched on patient and hospitalization characteristics using exact and Mahalanobis distance matching.

\section{Methods}

Unadjusted t-tests and adjusted multinomial logit regression analyses to compare the occurrence of readmissions and Emergency Department/Observation visits within 30 and 60-days post-discharge.

\section{Results}

No statistically significant differences in readmissions or Emergency Department /Observation visits between home health care and non-home health care patients were observed.

\section{Conclusions}

Home health care referral was not associated with lower rates of return to hospital within 30 and 60 days in this US sample matched on patient and clinical condition characteristics. This result raises the question of why home health care services did not produce evidence of lower post-discharge return to hospital rates. Focused attention by home health care programs on strategies to reduce readmissions is needed. 


\section{Keywords}

Effectiveness, Home health care, Readmission, Emergency Department visits, Utilization

\section{What is already known}

- Home health care services provide a bridge from hospital care to home-based recovery.

- Results of observational studies and home health program implementation have produced mixed results concerning effectiveness in reducing post-discharge return to the hospital.

- Studies of home health care outcomes are often confounded by selection of patients with specific disease conditions and at high risk for readmission.

\section{What this paper adds}

- In a matched sample analysis, patients referred for post-discharge home health care services had similar rates of return to the hospital for readmission or ED visits as patients not referred for home health care.

- As health care policies and funding priorities evolve toward value-based care services within integrated care models, strategies to leverage the potential of home health services to reduce readmissions are urgently needed.

\section{Introduction}

With costs of healthcare rising globally, hospitals are faced with competing pressures to manage inpatient care costs while at the same time reducing readmissions (Kristensen et al., 2015). In response to these policy pressures, and to meet the increased demand for care of an aging and chronically ill population (World Health Organization, 2015), home health care (HHC) services have emerged in many international healthcare markets as a prominent component of the hospital to home care continuum and the most common bridge strategy to reduce length of stay and cost of hospital care while also minimizing readmissions (Alliance for Home Health Quality and Innovation, n.d.; Tao, Ellenbecker, Chen, Zhan, and Dalton, 2012). This paper examines the effectiveness of HHC in reducing readmissions in a large multi-site sample from the United States (US).

In the US, one in every 7 (Henry J Kaiser Family Foundation, 2019; United Health Foundation et al., n.d.) hospital patients is readmitted within 30 days after discharge, making repeat hospital admissions one of the most frequent and costly adverse outcomes of hospitalization. Readmission reduction is incentivized on the national level through federal programs like Accountable Care Organizations and Bundled Payments for Care Improvement, both designed to improve efficiency, quality, and coordination of care during the post-discharge transition and across the care continuum (CMS, 2018a, 2018b, 2018e). Integrated health systems participating in these programs frequently employ HHC for both cost and readmission reductions (C. D. Jones, Bowles, Richard, Boxer, and Masoudi, 2017).

Models for $\mathrm{HHC}$ delivery and funding comprise public and private care delivery entities and funding sources with varying levels of integration of home care and social services (Genet et al., 2011;Van Eenoo et al., 2018). In the US, HHC services are provided by either free-standing agencies or are part of integrated health care systems, consist of one or more visits conducted in the home to support the transition from hospital to home-based care, and may include skilled nursing care, physical and occupational therapy, social work, or case management/care coordination services (Landers et al., 2016). HHC professionals, primarily nurses, provide patients and families with surveillance of post-discharge progress, coordination and communication with follow-up care providers, education regarding disease condition, and symptom management, recovery expectations, and home 
management skills (CMS, 2018d, 2019a; Nelson and Pulley, 2015). HHC services are often bundled with other readmission-reduction interventions such as telephone contacts, and early ambulatory care visits

(Braet, Weltens, and Sermeus, 2016; Hansen, Young, Hinami, Leung, and Williams, 2011; Leppin et al., 2014).

Like hospitals, US HHC service providers are incentivized to minimize readmissions. Federal initiatives driving health care improvements have instituted public reporting requirements and payment penalties for excessive readmissions under several programs including CMS' Hospital Readmission Reduction Program (HRRP) and the Improving Medicare Post-Acute Care Transformation (IMPACT) Act (2014), which applied standardization of data across post-acute settings and created new quality measures, including potentially preventable readmissions CMS (2018e). CMS quality of patient care star ratings posted on the Home Health Compare website CMS (2019b) are determined in part by readmission rates which can affect HHC agency's client recruitment and both Medicare and commercial reimbursement.

Despite the promise of reducing readmissions, evidence of the effectiveness of HHC in reducing readmissions is mixed. Numerous studies have demonstrated a beneficial impact from implementation of home follow-up programs for post-discharge recovery and longer-term disease management, yet few tracked readmissions. In a meta-analysis of transitional care interventions and readmissions in congestive heart failure patients (Feltner et al., 2014), only two of forty-seven were randomized controlled trials of a home visiting intervention with readmission measured within 30-days post-hospital discharge, and only one of the two studies reported a reduction in readmissions for patients receiving home visiting (Naylor et al., 2004). Evidence from observational studies is mixed-some reported effectiveness of home visiting (de Mestral et al., 2019; O'Connor, Hanlon, Naylor, and Bowles, 2015; Xiao, Miller, Zafirau, Gorodeski, and Young, 2018), while others reported same or higher rates of readmission for patients receiving $\mathrm{HHC}$ than patients without $\mathrm{HHC}$ services post-discharge (Dong, Cursio, Qadir, Lindenauer, and Ruhnke, 2017; Martin et al., 2011; Riggs, Roberts, Aronow, and Younan, 2010; Sanford et al., 2014). For example, post-discharge readmission rates were higher with HHC services in studies of patients following pancreatectomy ( $\mathrm{HHC}=24.3 \%$ vs non-HHC $=19.8 \%$ within 30 days) (Sanford et al., 2014), abdominal surgery ( $\mathrm{HHC}=62 \%$ vs. non-HHC $=11 \%$ within 30 days) (Martin et al., 2011), joint replacement ( $\mathrm{HHC}=10.5 \%$ vs non- $\mathrm{HHC}=5.1 \%$ within 6 months) (Riggs et al., 2010), and community acquired pneumonia patients ( $\mathrm{HHC}=20.1 \%$ vs non- $\mathrm{HHC}=11.5 \%$ within 30 days) (Dong et al., 2017). However, nonrandomized observational studies can be skewed toward finding a positive association of $\mathrm{HHC}$ with readmissions because HHC services tend to be prioritized for high-risk patients.

In contrast to much of the extant literature, our study compares post-discharge utilization outcomes from referral to HHC to community-based care without home health care by matching HHC and non-HHC groups from a broader population of patients discharged from acute care hospitals, rather than specific conditions. Our observational study estimates the overall effectiveness of $\mathrm{HHC}$ in avoiding post-discharge return to the hospital for readmission, or emergency or observation visits without inpatient admission. We conducted this study by employing exact and Malahanobis distance matching on multi-site data collected from hospitals' administrative databases as well as data collected directly from patients and their discharging nurse to compare post-discharge utilization across a diverse set of patient and hospitalization characteristics, and health conditions.

\section{Theoretical framework}

Meleis' Transitions Theory (Meleis, 2000) provided the framework used to guide the design and selection of variables included in this study (See Table 1). In this framework, a transition involves the processes of adaptation to a maturational or situational change in life situation and/or condition. Hospital discharge is a situational transition about which considerable research has been conducted using Transitions Theory as the guiding theoretical framework (Meleis, 2010). The notion that $\mathrm{HHC}$ is a bridge strategy to facilitate transition from hospital to home aligns with Transitions Theory. The premise of Transitions Theory is that there are three groups 
of variables impacting the patient's pattern of response to the transition: nature (or properties) of the transition, transitions conditions, and nursing/health team therapeutics. In this study, "nature of transition" variables are factors related to the nature of the hospitalization, such as prior hospitalization and length of stay, that can affect the "pattern of response" following hospital discharge (return to the hospital for readmission or Emergency Department [ED] visits). "Transition conditions" are the personal characteristics that facilitate or inhibit successful transition; in this study the patient characteristics of interest include, for example, age, gender, and payer. "Therapeutics" includes actions of the nurse/health team to influence the transition and is represented in this study by the decision to discharge home with HHC services. Consistent with Transitions Theory, we hypothesize that discharge home with HHC services (nurse/health team therapeutics) will, after controlling for other hospitalization factors (the nature of transition) and patient characteristics (transition conditions), be associated with improved post-discharge outcomes (pattern of response), measured as readmissions and ED visits.

Table 1. Theoretical Framework and Empirical Measures.

\begin{tabular}{|c|c|c|c|c|}
\hline $\begin{array}{l}\text { Transitions } \\
\text { Theory } \\
\text { Concepts }\end{array}$ & $\begin{array}{l}\text { Nature of } \\
\text { Transitions }\end{array}$ & $\begin{array}{l}\text { Transition } \\
\text { Conditions: } \\
\text { Facilitators and } \\
\text { Inhibitors }\end{array}$ & Patterns of Response & $\begin{array}{l}\text { Nursing/Health } \\
\text { Team } \\
\text { Therapeutics }\end{array}$ \\
\hline $\begin{array}{l}\text { Discharge } \\
\text { Transition } \\
\text { Concepts } \\
\end{array}$ & $\begin{array}{l}\text { Discharge } \\
\text { transition to } \\
\mathrm{HHC}\end{array}$ & $\begin{array}{l}\text { Personal Facilitators } \\
\text { and Inhibitors }\end{array}$ & Post-discharge utilization & $\begin{array}{l}\text { Discharge with } \\
\text { HHHC }\end{array}$ \\
\hline Definitions & $\begin{array}{l}\text { Type, } \\
\text { properties } \\
\text { and pattern } \\
\text { of the } \\
\text { transition }\end{array}$ & $\begin{array}{l}\text { Patient characteristics } \\
\text { that can influence the } \\
\text { decision to refer to } \\
\text { HHC and can impact } \\
\text { post-discharge } \\
\text { outcomes }\end{array}$ & $\begin{array}{l}\text { Readmission or ED/Observation } \\
\text { visits after discharge }\end{array}$ & $\begin{array}{l}\text { Discharge } \\
\text { destination= } \\
\text { home with or } \\
\text { without HHC }\end{array}$ \\
\hline $\begin{array}{l}\text { Empirical } \\
\text { Measures }\end{array}$ & $\begin{array}{l}\text { - Length of } \\
\text { stay (days) } \\
\text { - Prior } \\
\text { hospitaliz- } \\
\text { ations ( } 30 \\
\text { and } 90 \text { days) } \\
\text {-ICU days }\end{array}$ & $\begin{array}{l}\text { Characteristics } \\
\text {-Age } \\
\text { - Race } \\
\text { - Ethnicity } \\
\text { - Gender } \\
\text { - Medical/Surgical } \\
\text { patient type } \\
\text { - Severity of Illness } \\
\text { - Major Diagnostic } \\
\text { Category } \\
\text { - Elixhauser } \\
\text { Comorbidity Index } \\
\text {-Payer } \\
\text { - Functional Status } \\
\text { (nurse reported) } \\
\text { - Lives alone } \\
\text {-Self-care ability } \\
\text { (patient reported) } \\
\text { - Social support } \\
\text { (patient reported) } \\
\text {-Propensity Score } \\
\text { - Hospital }\end{array}$ & $\begin{array}{l}\text { Post-discharge utilization of } \\
\text { readmissions and/or ED visits } \\
\text { within } 30 \text { and } 60 \text { days ( } 2= \\
\text { ED/Observation visit no } \\
\text { readmission, } 1=\text { readmission, } 0=\text { no } \\
\text { readmission/ED/Observation visit) }\end{array}$ & $\begin{array}{l}\text { Discharge } \\
\text { destination } \\
(1=\text { yes } / 0=\text { no })\end{array}$ \\
\hline
\end{tabular}




\section{Methods}

\section{Study design}

Using a matched case-control comparative design, this study was a secondary analysis of data from a multi-site randomized clinical trial (READI study (Weiss et al., 2019); NCT \# 01873118) evaluating the effectiveness of standardized discharge readiness protocols in reducing post-discharge return to the hospital through 30 and 60days. The READI study (subsequently referred to as the parent study) was conducted on adult medical-surgical units in 31 Magnet ${ }^{\circledR}$ hospitals in the United States (US) and 2 Magnet ${ }^{\circledR}$ hospitals in Saudi Arabia. Two units in each hospital were randomly assigned to implementation and control conditions. The US hospitals were distributed broadly across the US and equally represented community hospitals and academic medical centers, with bed sizes ranging from 180 to more than 1000 beds. Hospital units were primarily medical (39\%) and mixed medical-surgical units (42\%) (Bobay et al., 2015)(. A total sample of 144,868 inpatients discharged to home was accrued. Data were collected between September 2014 and March 2017.

\section{Sample}

The sample for this analysis included adults (18+ years), admitted to the hospital as inpatients and subsequently discharged from medical-surgical units with discharge destinations of home with or without HHC. We excluded patients from the 2 non-US hospitals and patients from 2 US hospitals not reporting discharges with HHC. We further restricted the sample to patients from implementation units from two of the four phases of the parent study (Weiss et al., 2019). It was critical to creating comparable HHC and Non-HHC groups that we limit the sample to include only patients who were exposed to the intervention, as the discharge readiness assessments that formed the intervention protocol may have affected discharge decisions about length of stay and referral to HHC services post-discharge. Some of the variables needed for our analysis (lives alone, functional status, selfcare ability, and expected amount of social support after discharge) were obtained directly from nurse assessment and patient self-report that were available only in the last 2 phases of the study and only from implementation unit (not control unit) patients.

The available sample (full sample $n=18,555)$ included patients discharged with $\mathrm{HHC}(n=3,579)$ and discharged without $\mathrm{HHC}(n=14,976)$. In order to compare patients with similar observed characteristics, we used a combination of exact and nearest-neighbor matching with replacement to match HHC patients to patients without $\mathrm{HHC}$ to create samples for comparison. Out of 3,579 patients referred to HHC, 2,767 (77.3\%) were matched successfully (matched $\mathrm{HHC}$ ) to 2,767 non-HHC referred patients (matched non-HHC).

\section{Study variables}

Table 1 presents the study variables aligned with theoretical framework constructs. Participating hospitals extracted data for the exposure, outcome, and patient characteristics variables from their electronic health records. Some patient characteristics were captured via patient and nurse surveys used in real-time within the parent study and were entered and merged with electronic data. Data were supplied to the study database in de-identified form.

We derived the exposure variable, discharge with $\mathrm{HHC}$, from the hospital discharge disposition code in the electronic health record as captured for mandatory reporting to state/federal databases (Discharge to home or self-care was coded as $0=$ discharge home without HHC; Discharged/transferred to home under care of organized home health service organization in anticipation of covered skilled care was coded as 1=discharge home with $\mathrm{HHC}$ ). Disposition codes do not specify the type of HHC services, timing of initiation of services, or number of visits ordered or completed.

The multinomial outcome variable was post-discharge return to hospital within 30 and 60 days measured from discharge from the index hospitalization, and coded as: $0=$ No readmissions or ED/Observation visits; 
$1=E D / O b s e r v a t i o n:$ defined as the occurrence of one or more visits to the ED or Observation visits without inpatient admission in the same encounter; and 2=Readmission: defined as the occurrence of one or more return visits to the hospital that included an inpatient stay with or without any ED/Observation visits.

Observation refers to return visits to the hospital that did not necessitate or result in an inpatient admission but patients are assigned a hospital bed (commonly coded as short stay $<23$ hours; outpatient-in-bed). The 60-day post-discharge windows was included because of variability in the length of services (number of visits) provided by HHC agencies that may extend beyond the 30-day period.

We included patient and hospitalization characteristics variables based on a review of the literature of predictors of HHC referral (Siclovan, 2018). These variables included age (in years); race (unknown, Native American/Alaskan, Asian American, Black/African American, Hawaiian/Pacific Islander, White[reference]); ethnicity (Hispanic, Non-Hispanic [reference], unknown); gender (female, male [reference]); payer (Medicare, Medicaid, uninsured, private insurance[reference], other payer type, and missing); length of hospital stay (defined as the number of midnights in hospital, categorized in quartiles); Major Diagnostic Category and Severity Of Illness (major, extreme, moderate, minor [reference], missing) from Diagnostic Related Groups coding; patient diagnosis type (medical/surgical); prior hospitalizations (an inpatient discharge within 30 days and 31-90 days prior to index admission); ICU days; Elixhauser Comorbidity Index (Elixhauser, Steiner, Harris, and Coffey, 2016).

Nurse-reported and patient-reported variables collected for the parent study were also used in the analysis as patient characteristics. Nurse-reported variables included the patient's functional status $(0=$ not independent/1=independent) and whether the patient lived alone ( $0=\mathrm{no} / 1=$ yes). Patient-reported variables included their self-care ability (0-10 scale) ("How well will you be able to perform your personal care (for example hygiene, bathing, toileting, and eating) at home"), and social support (0-10 scale) ("How much help will you have with your personal care after you go home?").

A propensity score for referral to $\mathrm{HHC}$ was calculated for each patient using a propensity score model derived using a separate sample of control group patients from the larger parent study (Supplemental Digital File: Methods and Table 1).

\section{Data analysis}

To create the matched samples, we used a combination of exact matching and nearest-neighbor matching using Mahalanobis distance measures to create balanced treatment $(\mathrm{HHC}$ ) and non-treatment (non-HHC) groups. We selected variables for exact (Guo and Fraser, 2015) and Mahalanobis distance matching (Guo and Fraser, 2015; Kantor, 2006; Warner, 2013) based on characteristics identified as significant and clinically meaningful predictors of $\mathrm{HHC}$ referral from a sample of control group patients from the parent study (Supplemental Digital File: Methods \& Table 1) as well as patient characteristics identified from the literature as indicative of receiving an $\mathrm{HHC}$ referral (Siclovan, 2018). Exact matching variables include hospital, gender, race (White/ non-White), medical-surgical patient type, prior hospitalization (30-day and 90-day combined), quartile of length-of-stay, Elixhauser Comorbidity Index score above (>7) or below ( $\leq 7)$ the median, lives alone, and functional status. Distance matching variables include age, patient-reported self-care ability and social support, and propensity score for HHC referral.

We tested the hypotheses that there would be a reduction in readmissions and ED/Observation visits with $\mathrm{HHC}$ compared to non-HHC using a two-sample test of proportions. As a sensitivity analysis, we calculated a multinomial logit regression to adjust for Major Diagnostic Category and for any patient control variables significantly different between HHC and non-HHC in the matched sample (Table 2).

Table 2. Full and Matched Sample Characteristics. 


\begin{tabular}{|c|c|c|c|c|c|c|}
\hline Characteristic & $\begin{array}{l}\text { Full Sample } \\
\text { (Available } \\
\text { for } \\
\text { Matching) }\end{array}$ & $\begin{array}{l}\text { Full } \\
\text { Sample } \\
\text { HHC } \\
\text { Patients }\end{array}$ & $\begin{array}{l}\text { Full Sample } \\
\text { Non-HHC } \\
\text { Patients }\end{array}$ & $\begin{array}{l}\text { Matched } \\
\text { HHC } \\
\text { Patients }\end{array}$ & $\begin{array}{l}\text { Matched } \\
\text { Non-HHC } \\
\text { Controls }\end{array}$ & $\begin{array}{l}\text { P- } \\
\text { Value }{ }^{\text {a Comparing }} \\
\text { Matched HHC/Non- } \\
\text { HHC }\end{array}$ \\
\hline & $N=18,555$ & $N=3,579$ & $N=14,976$ & $N=2,767$ & $N=2,767$ & \\
\hline \multicolumn{7}{|l|}{$\begin{array}{l}\text { Post-Discharge } \\
\text { Utilization }\end{array}$} \\
\hline \multicolumn{7}{|l|}{$\begin{array}{l}\text { 30-Day Utilization } \\
\neq\end{array}$} \\
\hline None & $\begin{array}{l}14,943 \\
(80.53)\end{array}$ & $\begin{array}{l}2,724 \\
(76.11)\end{array}$ & $\begin{array}{l}12,219 \\
(81.59)\end{array}$ & $\begin{array}{l}2,155 \\
(77.88)\end{array}$ & $\begin{array}{l}2,207 \\
(79.76)\end{array}$ & 0.108 \\
\hline $\mathrm{ED} / \mathrm{OBS}$ & $1,514(8.16)$ & $277(7.74)$ & $1,237(8.26)$ & $201(7.26)$ & $189(6.83)$ & 0.565 \\
\hline Readmission & $\begin{array}{l}2,098 \\
(11.31)\end{array}$ & $\begin{array}{l}578 \\
(16.15)\end{array}$ & $\begin{array}{l}1,520 \\
(10.15)\end{array}$ & $\begin{array}{l}411 \\
(14.85)\end{array}$ & $\begin{array}{l}371 \\
(13.41)\end{array}$ & 0.143 \\
\hline \multicolumn{7}{|l|}{$\begin{array}{l}\text { 60-Day Utilization } \\
\neq\end{array}$} \\
\hline None & $\begin{array}{l}13,582 \\
(73.20)\end{array}$ & $\begin{array}{l}2,443 \\
(68.26)\end{array}$ & $\begin{array}{l}11,139 \\
(74.38)\end{array}$ & $\begin{array}{l}1,957 \\
(70.73)\end{array}$ & $\begin{array}{l}1,982 \\
(71.63)\end{array}$ & 0.515 \\
\hline ED/OBS & $\begin{array}{l}1,943 \\
(10.47)\end{array}$ & $349(9.75)$ & $\begin{array}{l}1,594 \\
(10.64)\end{array}$ & $249(9.00)$ & $252(9.11)$ & 0.816 \\
\hline Readmission & $\begin{array}{l}3,030 \\
(16.33)\end{array}$ & $\begin{array}{l}787 \\
(21.99) \\
\end{array}$ & $\begin{array}{l}2,243 \\
(14.98) \\
\end{array}$ & $\begin{array}{l}561 \\
(20.27) \\
\end{array}$ & $\begin{array}{l}533 \\
(19.26) \\
\end{array}$ & 0.363 \\
\hline \multicolumn{7}{|c|}{$\begin{array}{l}\text { Home Health Care } \\
\text { Referral } \neq\end{array}$} \\
\hline No Referral & $\begin{array}{l}14,976 \\
(80.71)\end{array}$ & $0(0.00)$ & $\begin{array}{l}14,976 \\
(100.00)\end{array}$ & $0(0.00)$ & $\begin{array}{l}2,767 \\
(100.00)\end{array}$ & NA \\
\hline HHC Referral & $\begin{array}{l}3,579 \\
(19.29)\end{array}$ & $\begin{array}{l}3,579 \\
(100.00)\end{array}$ & $0(0.00)$ & $\begin{array}{l}2,767 \\
(100.00)\end{array}$ & $0(0.00)$ & NA \\
\hline \multicolumn{7}{|l|}{$\begin{array}{l}\text { Patient } \\
\text { Characteristics }\end{array}$} \\
\hline \multicolumn{7}{|l|}{ Gender $^{b, c} \neq$} \\
\hline Male & $\begin{array}{l}9,181 \\
(49.48)\end{array}$ & $\begin{array}{l}1,675 \\
(46.80)\end{array}$ & $\begin{array}{l}7,506 \\
(50.12)\end{array}$ & $\begin{array}{l}1,321 \\
(47.74)\end{array}$ & $\begin{array}{l}1,321 \\
(47.74)\end{array}$ & 1.000 \\
\hline Female & $\begin{array}{l}9,374 \\
(50.52)\end{array}$ & $\begin{array}{l}1,904 \\
(53.20)\end{array}$ & $\begin{array}{l}7,470 \\
(49.88)\end{array}$ & $\begin{array}{l}1,446 \\
(52.26)\end{array}$ & $\begin{array}{l}1,446 \\
(52.26)\end{array}$ & 1.000 \\
\hline \multicolumn{7}{|l|}{ Race $^{b} \neq$} \\
\hline White $^{c}$ & $\begin{array}{l}12,898 \\
(69.51)\end{array}$ & $\begin{array}{l}2,564 \\
(71.64)\end{array}$ & $\begin{array}{l}10,334 \\
(69.00)\end{array}$ & $\begin{array}{l}2,058 \\
(74.38)\end{array}$ & $\begin{array}{l}2,058 \\
(74.38)\end{array}$ & 1.000 \\
\hline $\begin{array}{l}\text { Black or African } \\
\text { American }\end{array}$ & $\begin{array}{l}2,610 \\
(14.07)\end{array}$ & $\begin{array}{l}454 \\
(12.69)\end{array}$ & $\begin{array}{l}2,156 \\
(14.40)\end{array}$ & $\begin{array}{l}305 \\
(11.02)\end{array}$ & $\begin{array}{l}288 \\
(10.41)\end{array}$ & 0.435 \\
\hline Asian & $504(2.72)$ & $103(2.88)$ & $401(2.68)$ & $64(2.31)$ & $72(2.60)$ & 0.437 \\
\hline $\begin{array}{l}\text { American Indian } \\
\text { or Alaska Native }\end{array}$ & $246(1.33)$ & $9(0.25)$ & $237(1.58)$ & $6(0.22)$ & $8(0.29)$ & 0.593 \\
\hline $\begin{array}{l}\text { Native } \\
\text { Hawaiian/Pacific } \\
\text { Islander }\end{array}$ & $43(0.23)$ & $18(0.50)$ & $25(0.17)$ & $17(0.61)$ & $8(0.29)$ & 0.049 \\
\hline Unknown & $\begin{array}{l}2,254 \\
(12.15)\end{array}$ & $\begin{array}{l}431 \\
(12.04)\end{array}$ & $\begin{array}{l}1,823 \\
(12.17)\end{array}$ & $\begin{array}{l}317 \\
(11.46)\end{array}$ & $\begin{array}{l}333 \\
(12.03)\end{array}$ & 0.479 \\
\hline
\end{tabular}




\begin{tabular}{|c|c|c|c|c|c|c|}
\hline $\begin{array}{l}\text { Medical/Surgical } \\
\text { Patient }{ }^{b, c} \neq\end{array}$ & & & & & & \\
\hline Medical & $\begin{array}{l}13,427 \\
(72.36)\end{array}$ & $\begin{array}{l}2,463 \\
(68.82)\end{array}$ & $\begin{array}{l}10,964 \\
(73.21)\end{array}$ & $\begin{array}{l}1,887 \\
(68.20)\end{array}$ & $\begin{array}{l}1,887 \\
(68.20)\end{array}$ & 1.000 \\
\hline Surgical & $\begin{array}{l}5,128 \\
(27.64)\end{array}$ & $\begin{array}{l}1,116 \\
(31.18)\end{array}$ & $\begin{array}{l}4,012 \\
(26.79)\end{array}$ & $\begin{array}{l}880 \\
(31.80)\end{array}$ & $\begin{array}{l}880 \\
(31.80)\end{array}$ & 1.000 \\
\hline $\begin{array}{l}\text { Prior } \\
\text { hospitalization } \\
\text { past } 90 \text { days }^{c} \neq\end{array}$ & $\begin{array}{l}4,078 \\
(21.98)\end{array}$ & $\begin{array}{l}1,161 \\
(32.44)\end{array}$ & $\begin{array}{l}2,917 \\
(19.48)\end{array}$ & $\begin{array}{l}775 \\
(28.01)\end{array}$ & $\begin{array}{l}775 \\
(28.01)\end{array}$ & 1.000 \\
\hline $\begin{array}{l}\text { Prior } \\
\text { hospitalization 0- } \\
30 \text { days }^{b}\end{array}$ & $\begin{array}{l}2,553 \\
(13.76)\end{array}$ & $\begin{array}{l}773 \\
(21.60)\end{array}$ & $\begin{array}{l}1,780 \\
(11.89)\end{array}$ & $\begin{array}{l}538 \\
(19.44)\end{array}$ & $\begin{array}{l}503 \\
(18.18)\end{array}$ & 0.230 \\
\hline $\begin{array}{l}\text { Prior } \\
\text { hospitalization 31- } \\
90 \text { days }^{\text {b }}\end{array}$ & $1,525(8.22)$ & $\begin{array}{l}388 \\
(10.84)\end{array}$ & $1,137(7.59)$ & $237(8.57)$ & $272(9.83)$ & 0.104 \\
\hline $\begin{array}{l}\text { Total Length of } \\
\text { Stay }{ }^{b} \S\end{array}$ & $4.34(3.91)$ & $\begin{array}{l}6.31 \\
(5.35) \\
\end{array}$ & $3.87(3.31)$ & $\begin{array}{l}6.24 \\
(5.43) \\
\end{array}$ & $5.87(4.33)$ & 0.007 \\
\hline $\begin{array}{l}\text { Lowest } \\
\text { Quartile }{ }^{c} \neq\end{array}$ & $\begin{array}{l}6,374 \\
(34.35)\end{array}$ & $\begin{array}{l}577 \\
(16.12)\end{array}$ & $\begin{array}{l}5,797 \\
(38.71)\end{array}$ & $\begin{array}{l}478 \\
(17.28)\end{array}$ & $\begin{array}{l}478 \\
(17.28)\end{array}$ & 1.000 \\
\hline 2nd Quartile ${ }^{c} \neq$ & $\begin{array}{l}3,537 \\
(19.06)\end{array}$ & $\begin{array}{l}517 \\
(14.45)\end{array}$ & $\begin{array}{l}3,020 \\
(20.17)\end{array}$ & $\begin{array}{l}381 \\
(13.77)\end{array}$ & $\begin{array}{l}381 \\
(13.77)\end{array}$ & 1.000 \\
\hline 3rd Quartile ${ }^{c} \neq$ & $\begin{array}{l}4,260 \\
(22.96) \\
\end{array}$ & $\begin{array}{l}843 \\
(23.55) \\
\end{array}$ & \begin{tabular}{|l|}
3,417 \\
$(22.82)$ \\
\end{tabular} & $\begin{array}{l}647 \\
(23.38) \\
\end{array}$ & $\begin{array}{l}647 \\
(23.38) \\
\end{array}$ & 1.000 \\
\hline Highest Quartile ${ }^{c}$ & $\begin{array}{l}4,384 \\
(23.63) \\
\end{array}$ & $\begin{array}{l}1,642 \\
(45.88) \\
\end{array}$ & $\begin{array}{l}2,742 \\
(18.31) \\
\end{array}$ & $\begin{array}{l}1,261 \\
(45.57) \\
\end{array}$ & $\begin{array}{l}1,261 \\
(45.57) \\
\end{array}$ & 1.000 \\
\hline \multicolumn{7}{|l|}{$\begin{array}{l}\text { Elixhauser } \\
\text { Comorbidity } \\
\text { Index }{ }^{\mathrm{b}} \S\end{array}$} \\
\hline $\operatorname{Low}(\leq 7)^{c} \neq$ & $\begin{array}{l}10,064 \\
(54.24)\end{array}$ & $\begin{array}{l}1,783 \\
(49.82)\end{array}$ & $\begin{array}{l}8,281 \\
(55.30)\end{array}$ & $\begin{array}{l}1,401 \\
(50.63)\end{array}$ & $\begin{array}{l}1,401 \\
(50.63)\end{array}$ & 1.000 \\
\hline $\operatorname{High}(>7)^{\mathrm{c}} \neq$ & $\begin{array}{l}8,491 \\
(45.76)\end{array}$ & $\begin{array}{l}1,796 \\
(50.18)\end{array}$ & $\begin{array}{l}6,695 \\
(44.70)\end{array}$ & $\begin{array}{l}1,366 \\
(49.37)\end{array}$ & $\begin{array}{l}1,366 \\
(49.37)\end{array}$ & 1.000 \\
\hline Lives Alone ${ }^{c} \neq$ & $\begin{array}{l}3,185 \\
(17.17)\end{array}$ & $\begin{array}{l}695 \\
(19.42)\end{array}$ & $\begin{array}{l}2,490 \\
(16.63)\end{array}$ & $\begin{array}{l}444 \\
(16.05)\end{array}$ & $\begin{array}{l}444 \\
(16.05)\end{array}$ & 1.000 \\
\hline $\begin{array}{l}\text { Functional status } \\
\text { (independent) }{ }^{c} \neq\end{array}$ & $\begin{array}{l}16,763 \\
(90.34)\end{array}$ & $\begin{array}{l}2,758 \\
(77.06)\end{array}$ & $\begin{array}{l}14,005 \\
(93.52)\end{array}$ & $\begin{array}{l}2,416 \\
(87.31)\end{array}$ & $\begin{array}{l}2,416 \\
(87.31)\end{array}$ & 1.000 \\
\hline Age $^{b, d} \S$ & $\begin{array}{l}59.32 \\
(17.21)\end{array}$ & $\begin{array}{l}67.99 \\
(14.93)\end{array}$ & $\begin{array}{l}57.25 \\
(17.07)\end{array}$ & $\begin{array}{l}67.26 \\
(14.82)\end{array}$ & $\begin{array}{l}64.01 \\
(14.32)\end{array}$ & $0.000^{\wedge \wedge \wedge}$ \\
\hline $\begin{array}{l}\text { Self-care Ability } \\
\text { (Patient- } \\
\text { reported) }{ }^{\mathrm{d}} \S\end{array}$ & $8.98(1.71)$ & $\begin{array}{l}8.39 \\
(2.06)\end{array}$ & $9.12(1.58)$ & $\begin{array}{l}8.54 \\
(1.91)\end{array}$ & $9.03(1.47)$ & $0.000^{\wedge \wedge \wedge}$ \\
\hline $\begin{array}{l}\text { Social Support } \\
\text { (Patient- } \\
\text { reported) }{ }^{d} \S \\
\end{array}$ & $8.15(3.04)$ & $\begin{array}{l}8.26 \\
(2.70)\end{array}$ & $8.13(3.11)$ & $\begin{array}{l}8.29 \\
(2.69)\end{array}$ & $8.66(2.39)$ & $0.000^{\wedge \wedge \wedge}$ \\
\hline $\begin{array}{l}\text { HHC Propensity } \\
\text { Score }{ }^{d} \S\end{array}$ & $0.19(0.17)$ & $\begin{array}{l}0.35 \\
(0.20) \\
\end{array}$ & $0.15(0.14)$ & $\begin{array}{l}0.33 \\
(0.20) \\
\end{array}$ & $0.28(0.18)$ & $0.000^{\wedge \wedge \wedge}$ \\
\hline ICU Days b $§$ & $0.60(1.88)$ & $\begin{array}{l}1.16 \\
(2.88) \\
\end{array}$ & $0.47(1.52)$ & $\begin{array}{l}1.19 \\
(2.93) \\
\end{array}$ & $0.96(2.02)$ & $0.001^{\wedge}$ \\
\hline $\begin{array}{l}\text { Severity of } \\
\text { Illness }{ }^{b} \neq\end{array}$ & & & & & & \\
\hline
\end{tabular}




\begin{tabular}{|c|c|c|c|c|c|c|}
\hline Minor & $\begin{array}{l}2,358 \\
(12.71 \%)\end{array}$ & $\begin{array}{l}152 \\
(4.25 \%)\end{array}$ & $\begin{array}{l}2,206 \\
(14.73 \%)\end{array}$ & $\begin{array}{l}124 \\
(4.48 \%)\end{array}$ & $\begin{array}{l}178 \\
(6.43 \%)\end{array}$ & 0.001 \\
\hline Moderate & $\begin{array}{l}5,723 \\
(30.84 \%)\end{array}$ & $\begin{array}{l}777 \\
(21.71 \%)\end{array}$ & $\begin{array}{l}4,946 \\
\text { (33.03\%) }\end{array}$ & $\begin{array}{l}606 \\
(21.90 \%)\end{array}$ & $\begin{array}{l}671 \\
(24.25 \%)\end{array}$ & 0.052 \\
\hline Major & $\begin{array}{l}5,734 \\
(30.90 \%)\end{array}$ & $\begin{array}{l}1,481 \\
(41.38 \%)\end{array}$ & $\begin{array}{l}4,253 \\
(28.40 \%)\end{array}$ & $\begin{array}{l}1,158 \\
(41.85 \%)\end{array}$ & $\begin{array}{l}1,090 \\
(39.39 \%)\end{array}$ & 0.068 \\
\hline Extreme & $\begin{array}{l}1,060 \\
(5.71 \%)\end{array}$ & $\begin{array}{l}435 \\
(12.15 \%)\end{array}$ & $625(4.17 \%)$ & $\begin{array}{l}323 \\
(11.67 \%)\end{array}$ & $\begin{array}{l}264 \\
(9.54 \%) \\
\end{array}$ & 0.013 \\
\hline Unknown & $\begin{array}{l}3,680 \\
(19.83 \%)\end{array}$ & $\begin{array}{l}734 \\
(20.51 \%)\end{array}$ & $\begin{array}{l}2,946 \\
(19.67 \%)\end{array}$ & $\begin{array}{l}556 \\
(20.09 \%)\end{array}$ & $\begin{array}{l}564 \\
(20.38 \%)\end{array}$ & 0.003 \\
\hline \multicolumn{7}{|l|}{ Payer Type ${ }^{b} \neq$} \\
\hline Private & $\begin{array}{l}5,386 \\
(29.03)\end{array}$ & $\begin{array}{l}711 \\
(19.87)\end{array}$ & $\begin{array}{l}4,675 \\
(31.22)\end{array}$ & $\begin{array}{l}599 \\
(21.65)\end{array}$ & $\begin{array}{l}736 \\
(26.60)\end{array}$ & $0.000^{\wedge \wedge \wedge}$ \\
\hline Medicare & $\begin{array}{l}7,848 \\
(42.30)\end{array}$ & $\begin{array}{l}2,124 \\
(59.35)\end{array}$ & $\begin{array}{l}5,724 \\
(38.22)\end{array}$ & $\begin{array}{l}1,633 \\
(59.02)\end{array}$ & $\begin{array}{l}1,379 \\
(49.84)\end{array}$ & $0.000^{\wedge \wedge \wedge}$ \\
\hline Medicaid & $\begin{array}{l}2,745 \\
(14.79)\end{array}$ & $330(9.22)$ & $\begin{array}{l}2,415 \\
(16.13) \\
\end{array}$ & $262(9.47)$ & $\begin{array}{l}316 \\
(11.42) \\
\end{array}$ & 0.020 \\
\hline Uninsured & $388(2.09)$ & $22(0.61)$ & $366(2.44)$ & $13(0.47)$ & $37(1.34)$ & $0.001^{\wedge}$ \\
\hline Other & $\begin{array}{l}2,188 \\
(11.79)\end{array}$ & $\begin{array}{l}392 \\
(10.95) \\
\end{array}$ & $\begin{array}{l}1,796 \\
(11.99) \\
\end{array}$ & $260(9.40)$ & $\begin{array}{l}299 \\
(10.81)\end{array}$ & 0.076 \\
\hline \multicolumn{7}{|l|}{ Ethnicity ${ }^{b} \neq$} \\
\hline Not Hispanic & $\begin{array}{l}16,514 \\
(89.00)\end{array}$ & $\begin{array}{l}3,279 \\
(91.62)\end{array}$ & $\begin{array}{l}13,235 \\
(88.37) \\
\end{array}$ & $\begin{array}{l}2,542 \\
(91.87)\end{array}$ & $\begin{array}{l}2,397 \\
(86.63)\end{array}$ & $0.000^{\wedge \wedge \wedge}$ \\
\hline Hispanic & $1,683(9.07)$ & $281(7.85)$ & $1,402(9.36)$ & $210(7.59)$ & $249(9.00)$ & 0.052 \\
\hline Unknown & $358(1.93)$ & $19(0.53)$ & $339(2.26)$ & $15(0.54)$ & $121(4.37)$ & $0.000^{\wedge \wedge \wedge}$ \\
\hline
\end{tabular}

Statistics presented: $\neq \mathrm{n}(\%) ; \S$ Mean (SD)

The full output including Major Diagnostic Categories and hospital fixed effects is reported in the Supplemental Digital File Table 2.

${ }^{a}$ Shown are $p$-values, the hat ${ }^{\wedge}$ symbols indicate Bonferroni-corrected significance: ${ }^{\wedge \wedge}$ means $0.1 \%,{ }^{\wedge}$ means $1 \%$, ^ means $5 \%$.

bIncluded in propensity score model

${ }^{\mathrm{C}}$ Exact matching variable

${ }^{\mathrm{d}}$ Mahalanobis distance matching variable

All statistical analyses were completed using Stata version 14 (StataCorp [computer program], 2015) with a $p<0.05$ level of significance.

Approvals

Approvals were obtained from the Institutional Review Board at Marquette University (primary IRB; \#HR2668), university IRBs of the research team members, and participating hospital IRBs or ethics review committees.

\section{Results}

Sample characteristics are presented in Table 2. The rate of discharge home with HHC was $19.3 \%(N=3,579)$ for the available (full) sample. The readmission rates for the full sample were $11.3 \%$ for 30 -day readmissions and $16.3 \%$ for 60 -day readmissions and the ED/Observation only visit rates were $8.2 \%$ within 30 -days and $10.5 \%$ within 60-days. 
In the unadjusted two-sample test of proportions using the matched $\mathrm{HHC} /$ non-HHC sample (Table 3), while the HHC group had slightly higher readmission rates of $1.5 \%$ for 30 -day readmissions $(14.9 \%$ [HHC]; $13.4 \%$ [non$\mathrm{HHC}]$ ) and $1.0 \%$ for 60 -day readmissions (20.3\% [HHC]; 19.3\% [non-HHC]), the differences between $\mathrm{HHC}$ and non-HHC rates were not statistically different $(p>0.05)$. ED/Observation rates were lower than readmission rates but there were no significant differences between groups at either 30 days (7.3\% [HHC]; $6.8 \%$ [non-HHC]) or $60-$ days (HHC: $9.0 \%[H H C] ; 9.1 \%[$ non-HHC]).

Table 3. Post-discharge utilization of matched samples with and without a Home Health Care Referral.

\begin{tabular}{|c|c|c|c|c|}
\hline & $\begin{array}{l}\text { 30-Day Post-Discharge } \\
\text { Utilization }\end{array}$ & & $\begin{array}{l}\text { 60-Day Post-Discharge } \\
\text { Utilization }\end{array}$ & \\
\hline & ED/Observation Visit & Readmission & ED/Observation Visit & Readmission \\
\hline & $\begin{array}{l}\text { Unadjusted Two-Sample } \\
\text { Test of Proportions }\end{array}$ & & & \\
\hline HHC Referral Group ${ }^{a}$ & 0.073 & 0.149 & 0.090 & 0.203 \\
\hline $\begin{array}{l}\text { Matched Control } \\
\text { Group }^{\text {a }}\end{array}$ & 0.068 & 0.134 & 0.091 & 0.193 \\
\hline \multirow{3}{*}{$\begin{array}{l}\text { Difference (HHC - } \\
\text { Control) }^{\mathrm{b}}\end{array}$} & 0.004 & 0.015 & -0.001 & 0.010 \\
\hline & $(0.0107)$ & $(0.0177)$ & $(0.0127)$ & $(0.020)$ \\
\hline & $\begin{array}{l}\text { Adjusted Multinomial } \\
\text { Logistic Regression }\end{array}$ & & & \\
\hline HHC Referral Group ${ }^{a}$ & 0.076 & 0.147 & 0.091 & 0.202 \\
\hline $\begin{array}{l}\text { Matched Control } \\
\text { Group }^{\text {a }}\end{array}$ & 0.066 & 0.135 & 0.090 & 0.193 \\
\hline \multirow{2}{*}{$\begin{array}{l}\text { Difference (HHC - } \\
\text { Control) }^{b}\end{array}$} & 0.010 & 0.012 & 0.002 & 0.009 \\
\hline & $(0.011)$ & $(0.021)$ & $(0.013)$ & $(0.023)$ \\
\hline \multicolumn{5}{|l|}{ Control Variables $^{c}$} \\
\hline \multirow[t]{2}{*}{ Age } & -0.0002 & $-0.0021 * * *$ & 0.0002 & $-0.0027^{* * *}$ \\
\hline & $(0.0006)$ & $(0.0006)$ & $(0.0006)$ & $(0.0007)$ \\
\hline \multirow{2}{*}{$\begin{array}{l}\text { High Elixhauser } \\
\text { Comorbidity Index (>7) }\end{array}$} & 0.0069 & $0.0365 * * *$ & 0.0079 & $0.0407 * * *$ \\
\hline & $(0.0085)$ & $(0.0122)$ & $(0.0085)$ & $(0.0139)$ \\
\hline \multirow{2}{*}{$\begin{array}{l}\text { Self-care Ability } \\
\text { (Patient-reported) }\end{array}$} & -0.0006 & -0.0009 & -0.0022 & -0.0006 \\
\hline & $(0.0032)$ & $(0.0033)$ & $(0.0030)$ & $(0.0043)$ \\
\hline \multirow{2}{*}{$\begin{array}{l}\text { Social Support } \\
\text { (Patient-reported) }\end{array}$} & 0.0016 & 0.0006 & -0.0002 & -0.0011 \\
\hline & $(0.0016)$ & $(0.0015)$ & $(0.0020)$ & $(0.0019)$ \\
\hline \multirow[t]{2}{*}{ HHC Propensity Score } & 0.0182 & $0.197^{* * *}$ & -0.0372 & $0.255^{* * *}$ \\
\hline & $(0.0347)$ & $(0.0539)$ & $(0.0498)$ & $(0.0639)$ \\
\hline \multirow[t]{2}{*}{ ICU Days } & -0.00145 & $-0.0050 *$ & 0.0015 & $-0.0103 * *$ \\
\hline & $(0.0023)$ & $(0.0029)$ & $(0.0034)$ & $(0.0049)$ \\
\hline \multirow[t]{2}{*}{ Hispanic Ethnicity } & 0.0097 & 0.0154 & 0.00335 & 0.0347 \\
\hline & $(0.0174)$ & $(0.0183)$ & $(0.0186)$ & $(0.0270)$ \\
\hline \multirow[t]{2}{*}{ Unknown Ethnicity } & $0.0581^{* *}$ & -0.0155 & 0.0208 & -0.0497 \\
\hline & $(0.0251)$ & $(0.0325)$ & $(0.0266)$ & $(0.0405)$ \\
\hline \multirow[t]{2}{*}{ Medicare Payer Type } & 0.0043 & 0.0079 & 0.0082 & 0.0262 \\
\hline & $(0.0127)$ & $(0.0273)$ & $(0.0168)$ & $(0.0236)$ \\
\hline
\end{tabular}




\begin{tabular}{|l|l|l|l|l|}
\hline Medicaid Payer Type & 0.0221 & 0.0271 & $0.0376^{*}$ & 0.0368 \\
\hline & $(0.0153)$ & $(0.0310)$ & $(0.0221)$ & $(0.0282)$ \\
\hline Uninsured Payer Type & 0.0347 & -0.0114 & 0.0331 & -0.0602 \\
\hline & $(0.0424)$ & $(0.0474)$ & $(0.0455)$ & $(0.0434)$ \\
\hline Other Payer Type & -0.0148 & 0.0012 & -0.0062 & 0.0049 \\
\hline & $(0.0121)$ & $(0.0281)$ & $(0.0136)$ & $(0.0266)$ \\
\hline Observations & 5,534 & 5,534 & 5,534 & 5,534 \\
\hline
\end{tabular}

$* * * p<0.001, * * p<0.01, * p<0.05$

The full output including Major Diagnostic Categories is reported in the Supplemental Digital File Table 3.

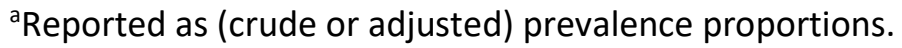

${ }^{\mathrm{b}}$ Reported as absolute percentage point difference in prevalence

'Reported as coefficients (marginal effects); standard errors in parentheses

We observed significant differences in 6 of the patient/hospitalization characteristics in our sample (age, patient-reported self-care ability and social support, ICU days, payer types [Private, Medicare, and Uninsured], and ethnicity [non-Hispanic, unknown]). The HHC matched sample was older (67.3 years [HHC]; 64.0 years [non$\mathrm{HHC}$ ], $p<0.001$ ), had more ICU days (1.2 days [HHC]; 1 days [non-HHC], $p=0.001$ ), fewer with private payers (21.7\% [HHC]; 26.6\% [non-HHC], $p<0.001)$, fewer uninsured $(0.5 \%$ [HHC]; 1.4\% [non-HHC], $p=0.001)$, more Medicare as payer (59.0\% [HHC]; 49.8\% [non-HHC], $p<0.001)$, lower patient-reported self-care ability $(8.5$ $[\mathrm{HHC}$; 9.0 [non-HHC], $p<.001)$ and social support ( 8.3 [HHC]; 8.7 [non-HHC], $p<0.001)$, and more patients with non-Hispanic (91.1\% [HHC]; 86.6\% [non-HHC], $p<0.001)$ and unknown ethnicity $(0.5 \%$ [HHC]; 4.4\% [non$\mathrm{HHC}], p<0.001)$.

The adjusted logit models (adjusting for Major Diagnostic Category, residual differences in the matched samples, and the propensity score) also indicated no significant differences between $\mathrm{HHC}$ and non-HHC for readmissions and ED/Observation visits within 30 and 60-days (Table 3).

Post-hoc power analyses indicated an adequate sample size for $80 \%$ power to detect a two percentage point difference between HHC and non-HHC groups at $p<0.05$ (UCLA: Statistical Consulting Group, n.d.). Because the observed differences in readmission and ED/Observation rates were less than two percentage points, we further explored our findings by stratifying patients into high and low comorbidity groups using the Elixhauser Comorbidity Index (Elixhauser et al., 2016) median score. In unadjusted and adjusted models, there were no statistically significant differences between $\mathrm{HHC}$ and non-HHC in readmissions or in ED/Observation visits in lowand high-comorbidity patients (See Table 4).

Table 4. Post-discharge utilization of matched samples with and without a Home Health Care referral stratified by low and high-comorbidity (Elixhauser Comorbidity Index $\leq 7$ [Low], $>7$ [High]).

\begin{tabular}{|c|c|c|c|c|}
\hline & $\begin{array}{l}\text { 30-Day Post-Discharge } \\
\text { Utilization }\end{array}$ & & $\begin{array}{l}\text { 60-Day Post-Discharge } \\
\text { Utilization }\end{array}$ & \\
\hline & ED/Observation Visit & Readmission & ED/Observation Visit & Readmission \\
\hline & $\begin{array}{l}\text { Unadjusted Two-Sample } \\
\text { Test of Proportions }\end{array}$ & & & \\
\hline $\begin{array}{l}\text { Low Elixhauser } \\
\text { Comorbidity Index } \\
(\leq 7)^{a}\end{array}$ & & & & \\
\hline HHC Referral Group ${ }^{b}$ & 0.070 & 0.127 & 0.086 & 0.171 \\
\hline $\begin{array}{l}\text { Matched Control } \\
\text { Group }^{\mathrm{b}}\end{array}$ & 0.054 & 0.110 & 0.077 & 0.165 \\
\hline
\end{tabular}




\begin{tabular}{|c|c|c|c|c|}
\hline Difference (HHC - & 0.016 & 0.017 & 0.009 & 0.006 \\
\hline & $(0.012)$ & $(0.023)$ & $(0.013)$ & $(0.024)$ \\
\hline \multicolumn{5}{|l|}{$\begin{array}{l}\text { High Elixhauser } \\
\text { Comorbidity Index } \\
(>7)^{a}\end{array}$} \\
\hline HHC Referral Group ${ }^{b}$ & 0.075 & 0.171 & 0.094 & 0.236 \\
\hline $\begin{array}{l}\text { Matched Control } \\
\text { Group }^{b}\end{array}$ & 0.083 & 0.159 & 0.105 & 0.221 \\
\hline \multirow[t]{3}{*}{$\begin{array}{l}\text { Difference (HHC - } \\
\text { Control) }^{c}\end{array}$} & -0.007 & 0.012 & -0.011 & 0.015 \\
\hline & $(0.013)$ & $(0.016)$ & $(0.016)$ & $(0.019)$ \\
\hline & $\begin{array}{l}\text { Adjusted Multinomial } \\
\text { Logistic Regression }\end{array}$ & & & \\
\hline \multicolumn{5}{|l|}{$\begin{array}{l}\text { Low Elixhauser } \\
\text { Comorbidity Index } \\
(\leq 7)^{a}\end{array}$} \\
\hline HHC Referral Group ${ }^{b}$ & 0.073 & 0.128 & 0.087 & 0.172 \\
\hline $\begin{array}{l}\text { Matched Control } \\
\text { Group }^{b}\end{array}$ & 0.052 & 0.109 & 0.076 & 0.163 \\
\hline \multirow[t]{2}{*}{$\begin{array}{l}\text { Difference (HHC - } \\
\text { Control) }^{c}\end{array}$} & 0.021 & 0.019 & 0.012 & 0.009 \\
\hline & $(0.013)$ & $(0.028)$ & $(0.014)$ & $(0.028)$ \\
\hline \multicolumn{5}{|l|}{$\begin{array}{l}\text { High Elixhauser } \\
\text { Comorbidity Index } \\
(>7)^{\mathrm{a}}\end{array}$} \\
\hline HHC Referral Group ${ }^{b}$ & 0.078 & 0.167 & 0.096 & 0.23 \\
\hline $\begin{array}{l}\text { Matched Control } \\
\text { Group }^{b}\end{array}$ & 0.080 & 0.162 & 0.104 & 0.22 \\
\hline \multirow[t]{2}{*}{$\begin{array}{l}\text { Difference (HHC - } \\
\text { Control) }^{c}\end{array}$} & -0.002 & 0.006 & -0.009 & 0.009 \\
\hline & $(0.013)$ & $(0.019)$ & $(0.016)$ & $(0.022)$ \\
\hline Observations & 5,534 & 5,534 & 5,534 & 5,534 \\
\hline
\end{tabular}

$* * * p<0.001, * * p<0.01, * p<0.05$

The full output including control variables (same as Table 3) and Major Diagnostic Categories is reported in the Supplemental Digital File Table 4.

aLow Elixhauser Comorbidity Index (<7) sample includes 2,802 (1,401 HHC patients and 1,401 non-HHC controls), and the high-Elixhauser sample includes 2,732 (1,366 HHC and 1,366 non-HHC).

${ }^{\mathrm{b}}$ Reported as (crude or adjusted) prevalence proportions.

${ }^{\mathrm{C}}$ Reported as absolute percentage point difference in prevalence, standard error in parentheses.

\section{Discussion}

This multi-hospital US study of a matched sample of patients with a broad range of clinical conditions did not show the reductions in readmission rates for $\mathrm{HHC}$ patients previously reported by some evaluations of disease specific home-visiting management programs and case-management interventions (Feltner et al., 2014; Maliakkal and Sun, 2014; Naylor, 2004). Conversely, adjusted readmission rates for HHC patients were consistently higher by up to 1.5 percentage points in all comparisons, albeit these differences were not 
statistically significant. In post-hoc stratified analyses, no association of readmission or ED/Observation rates with the HHC discharge status was evident among high and low co-morbidity patients.

Several possible factors may explain the lack of effectiveness of $\mathrm{HHC}$ in reducing readmissions. One explanation may be that, currently, US HHCs do not universally prioritize readmission avoidance as an organizational quality outcome. Post-acute care patients are only a portion of the patients served by HHC agencies; readmission avoidance may not be a priority if a HHC agency's volume of services generated by hospital discharges is low. Even though readmission is a prominent factor in home health quality reporting requirements in the US, the current payment structure does not always reward HHC agencies for preventing readmissions (Landers et al., 2016). Hospital penalties under HRRP are tied to readmission rates within 30 days of hospital discharge; therefore, the contribution of HHC to hospital system outcomes may not be a priority in health systems where hospital and post-acute care are not fully integrated.

Policy changes are on the horizon in the US that may influence HHC agencies to align their limited resources with reimbursement incentives for readmission avoidance. CMS' Home Health Care Value-Based Purchasing(HHVBP) Model, designed to penalize poorly performing HHC providers and reward top HHC performers, is increasingly shifting their provider performance evaluation metrics to outcome-based measures (including ED use and unplanned hospital admissions), with payment adjustments of up to $8 \%$ (upward or downward) by 2022 in the pilot states (CMS, 2018c; C. D. Jones et al., 2017).

Other possible factors that may interfere with HHC efforts to reduce readmissions include the HHC clinician's decision-making at the time of a HHC visit, including legal concerns. HHC visits may actually hasten returns to the hospital through early identification and/or cautionary escalation of problems to a higher-level care, counterbalancing efforts to avoid returns to the hospital. Patients are more likely to visit the ED on days the HHC nurse visits the home than on days without a nurse visit, a relationship that persists even among lowcomorbidity patients (A. Jones et al., 2018). Medico-legal concerns may also underlie this pattern in the US and other countries. When identifying a worsening clinical condition, $\mathrm{HHC}$ nurses face limited immediate treatment options and respond based on patient needs and nurse competencies for the situation (Andersson, Lindholm, Pettersson, and Jonasson, 2017). Without timely access to necessary resources or situation-specific guidelines, nurses may recommend returning to the hospital to prevent negative health outcomes occurring at home (A. Jones et al., 2018). Lastly, considering that patients receiving home health services tend to be sicker on average that patients who do not, reducing readmissions may not be a feasible performance achievement bar for home health services.

To achieve readmission avoidance goals, HHC organizational structures must be realigned internally and with partner providers and organizations to improve collaboration across care teams and venues of care. (Busetto and Ger Luijkz, 2015; Kodner and Spreeuwenberg, 2002) New funding mechanisms for integrated care programs are becoming available that recognize the need for coordination among mental health services, health systems, health plans, and post-acute care health services (CMS, 2019b). Creating integrated care services to meet the needs of medically complex patients will require new resources, new partners, and new skills for home care professionals.

The skill level of the HHC workforce is an important contributor to patient outcomes. Given the increasing complexity of patients discharged to home, the skill set required for effective HHC care is expanding. Continuing education programs such as $\mathrm{HHC}$ residency and nurse certification programs could offer mechanisms for building an expert home health nurse workforce. Though educational programs such as residency programs are effective in increasing the competency of HHC nurses, less than $5 \%$ of $\mathrm{HHC}$ agencies offer such courses due to a lack of preceptors and the burden of costs associated with implementation and maintenance of these programs (Landers et al., 2016; Pittman, Horton, Terry, and Bass, 2014). Certification is a mechanism for validation of a 
nurse's knowledge, skills, and abilities (Chappell et al., 2019); however, home health nursing specialty certification was retired by the American Nurse Credentialing Center in 2005. Certifications for specific aspects of home care are available in hospice and palliative care, care coordination and transition management, and infusion therapy. Despite logistical barriers, HHC agencies must find ways to implement innovative programs for their staff as well as initiate and support efforts to reinvigorate interest in certification among the staff.

\section{Strengths and limitations}

This study had several major strengths relative to the published literature. First, compared to other disease- or diagnosis specific studies, many of which were conducted in a single facility or a single health system, our study is the first to use a large geographically-diverse multi-site database of general medical-surgical patients. Second, in addition to administrative hospital databases, we had patient self-reported data on self-care ability and social support variables not commonly available in administrative datasets. Third, we used robust matching methods which allowed us to minimize confounding from selection of high-risk patients for HHC assignment that was present in previous studies. Therefore, our study likely contributes some of the most rigorous, generalizable, and accurate information on the issue of broad-scale effectiveness of HHC use in preventing hospital readmissions.

There were several limitations to the design in this secondary analysis study. First, as in any observational study where a randomized controlled trial is not feasible or ethical, our matched study findings might still be confounded by unobservable and unmeasured variables. In the analysis of 60-day readmissions, the longer follow-up period may have further confounded the results. We attempted to reduce bias from sicker patients, who are, on average, more likely to be assigned to receive home health services; to do so, we matched on a comprehensive set of patient characteristics. However, a number of potentially important patient characteristics (e.g., depression, self-rating of health, communication challenges, adherence to the prescribed treatment plans, patient cognitive status, and caregiver status) were not available in the data and therefore could not be used in the matching protocol. To the extent that any unobserved confounding may remain in the data set, finding similar readmission rates in the HHC and the non-HHC groups may actually be considered a success of home health care in preventing readmissions in this high-risk patient population. Because our results were robust when additional patient variables (not used in the matching protocol) were added as direct adjustors in the final matched readmission model, residual confounding from unobserved patient characteristics is likely small.

Second, we did not have data on patient deaths outside of the hospital. A recent study of 8 million Medicare beneficiaries showed an association between readmission reductions and increased post-discharge mortality (Wadhera et al., 2018), which means that HHC effectiveness must be evaluated with respect to both outcomes. Continued prioritization of $\mathrm{HHC}$ outcomes is needed to determine its effectiveness. Aligning and incentivizing initiatives for HHC (IMPACT Act, HHVBP) is one way to motivate improvement efforts, but will take time to demonstrate. This study occurred at a time when incentivizing changes were only beginning and was not of a sufficient length to identify any differences resulting from various payment structures. Future studies using integrated data systems may be able to overcome our limitations.

Third, the data set applied to only those patients returning home, and not patients who were transferred to skilled or long term care facilities. Patients were coded as HHC if they received a referral for $\mathrm{HHC}$ at the time of discharge. We did not collect information about the type of home health agency (free standing or within a health system), quality information about the agency, whether HHC services actually occurred, number of visits, and the purpose and content of the visits. Lastly, we used same-hospital readmission and ED visits. Samehospital readmissions may not accurately represent all hospital readmissions (Gonzalez, Shih, Dimick, and Ghaferi, 2013). Our results were derived from a sample of patients discharged home within the US healthcare 
system and therefore may not represent outcomes in countries with different clinical care approaches and funding priorities for delivery of post-hospitalization home health care services.

\section{Conclusion}

Quality measurement and the business case for the value of HHC services for transitional care require demonstration of effectiveness in reducing return to the hospital post-discharge. In this study of comparable samples of US patients, HHC did not reduce readmissions or ED visits post-discharge. With $\mathrm{HHC}$ reimbursement becoming increasingly linked to quality measure reporting and contributing to health system performance outcomes in the US and internationally, prioritization of readmission avoidance as an HHC organizational outcome is imperative for demonstrating value to the healthcare system. New models aligning $\mathrm{HHC}$ with the larger health system will need to be developed, with targeted integrated care processes, payment models, and clinical staff with appropriate skills, resources, and decision support to improve their effectiveness in reducing readmission rates in the types of patients referred to HHC.

\section{Funding sources}

The READI study (Weiss, PI), the data source for this analysis, was commissioned by the American Nurse Credentialing Center (ANCC) following a competitive application process. Participating hospitals paid a fee to the ANCC to participate. The ANCC had no role in the design and conduct of the study; collection, management, analysis, and interpretation of the data; preparation, review, or approval of the manuscript; and the decision to submit the manuscript for publication.

\section{CRediT authorship contribution statement}

All authors have made substantial contributions to all of the following: (1) the conception and design of the study, or acquisition of data, or analysis and interpretation of data, (2) drafting the article or revising it critically for important intellectual content, (3) final approval of the version to be submitted.

\section{Declaration of Competing Interest}

None

\section{References}

Alliance for Home Health Quality and Innovation, Alliance for Home Health Quality and Innovation. (n.d.). Home health care data \& readmissions. Retrieved from http://www.ahhqi.org/images/pdf/what-is-hhc-datareadmissions.pdf

Andersson et al., 2017. H. Andersson, M. Lindholm, M. Pettersson, L.L. Jonasson. Nurses' competencies in home healthcare: an interview study. BMC Nurs., 16 (2017), p. 65, 10.1186/s12912-017-0264-9

Bobay et al., 2015. KL Bobay, S Bahr, ME Weiss, R Hughes, L Costa. Models of discharge care in Magnet hospitals. Journal of Nursing Administration, 45 (10) (2015), pp. 485-

491, 10.1097/NNA.0000000000000239

Braet et al., 2016. A. Braet, C. Weltens, W. Sermeus. Effectiveness of discharge interventions from hospital to home on hospital readmissions: a systematic review. JBI Database Syst. Rev Implement. Rep., 14 (2) (2016), pp. 106-173, 10.11124/jbisrir-2016-2381

Busetto and Ger Luijkz, 2015. L. Busetto, K. Ger Luijkz. Intervention types and outcomes of integrated care for diabetes mellitus type 2: a systematic review. J. Eval. Clin. Pract., 22 (3) (2015), pp. 299-310

Chappell et al., 2019. K. Chappell, D. Jeong, E.C. R., V. Lundmark, D. Kendall-Gallagher, E. Salt, S. Kitto. Constructing a sensitizing definition of a certification in nursing for research purposes: a hybrid 
methodology using consensus-building approach. J. Nurs. Adm., 49 (1) (2019), pp. $12-$ $18,10.1097 /$ NNA.0000000000000701

CMS 2018a. CMS. (2018a, August 31, 2018). Accountable Care Organizations (ACOs): General Information.

Retrieved from https://innovation.cms.gov/initiatives/aco/

CMS 2018b. CMS. (2018b). Home Health Star Ratings. Retrieved from https://www.cms.gov/Medicare/QualityInitiatives-Patient-Assessment-Instruments/HomeHealthQualityInits/HHQIHomeHealthStarRatings.html

CMS 2018c. CMS. (2018c). Home Health Value-Based Purchasing Model. Retrieved from https://innovation.cms.gov/initiatives/home-health-value-based-purchasing-model

CMS 2018d. CMS. (2018d). Home helath Quality Reporting Requirements. Retrieved from https://www.cms.gov/Medicare/Quality-Initiatives-Patient-AssessmentInstruments/HomeHealthQualityInits/Home-Health-Quality-Reporting-Requirements.html

CMS 2018e. CMS. (2018e). IMPACT Act Spotlights and Announcements. Retrieved from https://www.cms.gov/Medicare/Quality-Initiatives-Patient-Assessment-Instruments/Post-AcuteCare-Quality-Initiatives/IMPACT-Act-of-2014/Spotlights-and-Announcements-.html

CMS 2019a. CMS. (2019a, April 10, 2019). Home health quality reporting program. Retrieved from https://www.cms.gov/medicare/quality-initiatives-patient-assessmentinstruments/homehealthqualityinits/index.html

CMS 2019b. CMS. (2019b). What is Home Health Compare. Retrieved from https://www.medicare.gov/HomeHealthCompare/About/What-Is-HHC.html

de Mestral et al., 2019. C. de Mestral, A. Kayssi, M. Al-Omran, K. Salata, M. Anas Hussain, G. Roche-Nagle. Home care nursing after elective vascular surgery: an opportunity to reduce emergency department visits and hosptial readmission. BMJ Qual. Safety (2019), 10.1136/bmjqs-2018-009161

Dong et al., 2017. T. Dong, J.F. Cursio, S. Qadir, P.K. Lindenauer, G.W. Ruhnke. Discharge disposition as an independent predictor of readmission among patients hospitalised for community-acquired pneumonia. Int. J. Clin. Practice, 71 (3-4) (2017)

Elixhauser et al., 2016. Elixhauser, A., Steiner, C., Harris, D. R., \& Coffey, R. M. (2016). Concept: Elixhauser comorbidity index. Retrieved from http://mchpappserv.cpe.umanitoba.ca/viewConcept.php?printer=Y\&conceptID=1436

Feltner et al., 2014. C. Feltner, C.D. Jones, C.W. Cene, Z. Zheng, C.A. Sueta, E.J.L. Coker-Schwimmer, D.E. Jonas. Transitional care interventions to prevent readmissions for persons with heart failure: a systematic review and meta-analysis. Ann. Intern. Med., 160 (11) (2014), pp. 774-784

Genet et al., 2011.

N. Genet, W.G. Boerma, D.S. Kringos, A. Bouman, A.L. Francke, C. Fagerström, M.G. Melchiorre, C. Greco ,W. Devillé. Home care in Europe: a systematic literature review. BMC health services research, 11 (2011), p. 207. https://doi.org/10.1186/1472-6963-11-207

Gonzalez et al., 2013. A. Gonzalez, T. Shih, J.B. Dimick, A.A. Ghaferi. Does same-hospital readmission rate correlate with all-hospital readmission rate? J. Am. Coll.

Surg., 217 (3) (2013), 10.1016/j.jamcollsurg.2013.07.240 Supplement, S105

Guo and Fraser, 2015. S. Guo, M. Fraser. Propensity Score Analysis: Statistical Methods and Applications. (2nd ed.), Sage publications, Thousand Oaks, CA (2015)

Hansen et al., 2011. L.O. Hansen, R.S. Young, K. Hinami, A. Leung, M.V. Williams. Interventions to reduce 30-day rehospitalization: a systematic review. Ann. Intern. Med., 155 (8) (2011), pp. 520-528

Henry, 2019. Henry J, Kaiser Family Foundation. (2019). A Dozen Facts Abou Medicare Advantage in 2019. Retrieved from https://www.kff.org/medicare/issue-brief/a-dozen-facts-about-medicare-advantage-in2019/ 
Jones et al., 2018. A. Jones, C. Schumacher, S.E. Bronskill, M.A. Campitelli, J.W. Poss, H. Seow, A.P. Costa. The association between home care visits and same-day emergency department use: a case-crossover study. CMAJ, 190 (17) (2018), pp. E525-E531, 10.1503/cmaj.170892

Jones et al., 2017. C.D. Jones, K.H. Bowles, A. Richard, R.S. Boxer, F.A. Masoudi. High-value home health care for patients with heart failure: an opportunity to optimize transitions from hospital to home. Circulation, 10 (5) (2017), pp. 1-5, 10.1161/CIRCOUTCOMES.117.003676

Kantor, 2006. D. Kantor. MAHAPICK: Stata module to select matching observations based on a Mahalanobis distance measure (Version Revised 15 Nov 2012). Boston College Depart. Econ. (2006) S456703

Kodner and Spreeuwenberg, 2002. D.L. Kodner, C. Spreeuwenberg. Integrated care: meaning, logic, applications, and implications-a discussion paper. Int. J. Integr. Care, 2 (14) (2002), pp. 1-6

Landers et al., 2016. Landers, S., Madigan, E., Leff, B., Rosati, R. J., McCann, B. A., Hornbake, R., ... Breese, E. (2016). The future of home health care: a strategic framework for optimizing value. Home Health Care Manag Pract, 28(4), 262-278. doi:10.1177/1084822316666368

Kristensen et al., 2015. SR Kristensen, M Bech, W Quentin. A roadmap for comparing readmission policies with application to Denmark, England, Germany and the United States, 119, Health Policy (Amsterdam, Netherlands (2015 Mar), pp. 264-273, 10.1016/j.healthpol.2014.12.009

Leppin et al., 2014. A.L. Leppin, M.R. Gionfriddo, M. Kessler, J.P. Brito, F.S. Mair, K. Gallacher, V.M. Montori. Preventing 30-day hospital readmissions: a systematic review and meta-analysis of randomized trials. JAMA Intern. Med., 174 (7) (2014), pp. 1095-1107, 10.1001/jamainternmed.2014.1608

Maliakkal and Sun, 2014. Maliakkal, A. V., \& Sun, A. Z. (2014). Home care program reduces hospital readmissions in patients with Congestive Heart Failure and improves other associated indicators of health. Home Health Care Management \& Practice, 26(4), 191-197. doi:10.1177/1084822314527763

Martin et al., 2011. R.C.G. Martin, R. Brown, L. Puffer, S. Block, G. Callender, A. Quillo, K.M. McMasters. Readmission rates after abdominal surgery: the role of surgeon, primary caregiver, homehealth, and subacute rehab. Ann. Surg., 254 (4) (2011), pp. 591-597

Meleis et al., 2000. A.I. Meleis, L.M. Sawyer, E. Im, D.K. Hilfinger Messias, K. Schumacher. Experiencing transitions: an emerging middle-range theory. Adv. Nurs. Sci., 23 (1) (2000), pp. 12-28

Naylor et al., 2004. M.D. Naylor, D.A. Brooten, R.L. Campbell, G. Maislin, K.M. McCauley, J.S. Schwartz. Transitional care of older adults hospitalized with heart failure: A randomized controlled trial. J. Am. Geriatr. Soc., 52 (5) (2004), pp. 675-684

Nelson and Pulley, 2015. J.M. Nelson, A.L. Pulley. Transitional care can reduce hospital readmissions: A bundle of actvities linked to transitional care principles can reduce both short-and lont-term readmission risk. American Nurs. Today, 10 (4) (2015), pp. 1-8

O'Connor et al., 2015. M. O'Connor, A. Hanlon, M.D. Naylor, K.H. Bowles. The impact of home health length of stay and number of skilled nursing visits on hospitalization among medicare-reimbursed skilled home health beneficiaries. Res. Nurs. Health, 38 (4) (2015), pp. 257-267, 10.1002/nur.21665

Pittman et al., 2014. P. Pittman, K. Horton, M. Terry, E. Bass. Residency programs for home health and hospice nurses: Prevalence, barriers, and potential policy responses. Home Health Care Manage.

Pract., 26 (2) (2014), pp. 86-91. doi:https://doi.org/10.1177\%2F1084822313511457

Riggs et al., 2010. R.V. Riggs, P.S. Roberts, H. Aronow, T. Younan. Joint replacement and hip fracture readmission rates: impact of discharge destination. Phys. Med. Rehabil., 2 (2010), pp. 806-810

Sanford et al., 2014.

D.E. Sanford, M.A. Olsen, K.M. Bommarito, M. Shah, R.C. Fields, W.G. Hawkins, D.C. Linehan. Association of discharge home with home health care and 30-day readmission after pancreatectomy. J. Am. Coll. Surg., 219 (5) (2014), 10.1016/j.jamcollsurg.2014.07.008. 875-886 e871

Siclovan, 2018. D.M. Siclovan. The effectiveness of home health care for reducing readmissions: an integrative review. Home Health Care Serv. Q., 37 (3) (2018), pp. 187-210, 10.1080/01621424.2018.1472702 
StataCorp [computer program] 2015. StataCorp [computer program]. Stata Statistical Software (Version 14). StataCorp LP, TX (2015). College Station

Tao et al., 2012. H. Tao, C.H. Ellenbecker, J. Chen, L. Zhan, J. Dalton. The Influence of social environmental factors on rehospitalization among patients receiving home health care services. Adv. Nurs. Sci., 35 (4) (2012), pp. 346-358, 10.1097/ANS.0b013e318271d2ad

UCLA: Statistical Consulting Group, UCLA: Statistical Consulting Group. (n.d.). Powerlog: Command to compute power for logistic regression. Retrieved from https://stats.idre.ucla.edu/stata/dae/logistic-regressionpower-analysis/

United Health Foundation et al., United Health Foundation. (n.d.). America's Health Rankings. Retrieved from https://www.americashealthrankings.org/explore/senior/measure/hospital_readmissions_sr/state /ALL

Van Eenoo et al., 2018. L. Van Eenoo, H. van der Roest, G. Onder, H. Finne-Soveri, V. GarmsHomolova, P.V. Jonsson, S. Draisma, H. van Hout, A. Declercq. Organizational home care models across Europe: A cross sectional study. International Journal of Nursing Studies, 77 (2018), pp. 3945, 10.1016/j.ijnurstu.2017.09.013

Wadhera et al., 2018. R.K. Wadhera, K.E. Joynt Maddox, J.H. Wasfy, S. Haneuse, C. Shen, R.W Yeh. Association of the hospital readmissions reductions program with mortality among medicare beneficiaries hospitalized for heart failure, acute myocardial infarction, and pneumonia. JAMA, 320 (24) (2018), pp. 2542-2552, 10.1001/jama.2018.19232

Warner, 2013. R. Warner. Applied Statistics: From Bivariate Through Multivariate Techniques. (2nd ed.), Sage Publications, Thousand Oaks, CA (2013)

Weiss et al., 2019.

ME Weiss, O Yakusheva, KL Bobay, L Costa, RG Hughes, S Nuccio, M Hamilton, S Bahr, D Siclovan, J Bang. Effect of implementing discharge readiness assessment in adult medical-surgical units on 30-day return to hospital: The READI randomized clinical trial. JAMA Network Open, 2 (1) (2019), p. e187387, 10.1001/jamanetworkopen.2018.7387. https://jamanetwork.com/journals/jamanetworkopen/fullarticle/2722575

Xiao et al., 2018. R. Xiao, J.A. Miller, W.J. Zafirau, E.Z. Gorodeski, J.B. Young. Impact of home health care on health care resource utilization following hospital discharge: a cohort study. Am. J. Med., 131 (4) (2018), 10.1016/j.amjmed.2017.11.010. 395-407.e335

World Health Organization, 2015. World Health Organization (2015). World report on ageing and health. Geneva, Switzerland: World Health Organization. Available at: https://apps.who.int/iris/bitstream/handle/10665/186463/9789240694811_eng.pdf;jsessionid=AED 196F6CCBCB9F8690DFC5DAA901668? sequence $=1$ 